\title{
openheart Increased levels of sST2 in patients with mitral annulus disjunction and ventricular arrhythmias
}

\author{
Esther Scheirlynck, ${ }^{0,2,3}$ Lars A Dejgaard, ${ }^{2,4}$ Eystein Skjølsvik, ${ }^{2,4}$ Oyvind H Lie, ${ }^{2,4}$ \\ Andreea Motoc, ${ }^{3}$ Einar Hopp, ${ }^{5,6}$ Kaoru Tanaka, ${ }^{3,7}$ T Ueland, ${ }^{4,8}$ Margareth Ribe, ${ }^{2}$ \\ Carlos Collet, ${ }^{3,9}$ Thor Edvardsen, ${ }^{2,4}$ Steven Droogmans, ${ }^{1,3}$ Bernard Cosyns, ${ }^{1,3}$ \\ Kristina H Haugaa ${ }^{2,4}$
}

\begin{abstract}
- Additional material is published online only. To view please visit the journal online (http://dx.doi.org/10.1136/ openhrt-2019-001016).

To cite: Scheirlynck E, Dejgaard LA, Skjølsvik E, et al. Increased levels of SST2 in patients with mitral annulus disjunction and ventricular arrhythmias. Open Heart 2019;6:e001016. doi:10.1136/ openhrt-2019-001016
\end{abstract}

Received 15 January 2019 Revised 15 March 2019 Accepted 1 April 2019

\section{Check for updates}

(C) Author(s) (or their employer(s)) 2019. Re-use permitted under CC BY-NC. No commercial re-use. See rights and permissions. Published by BMJ.

For numbered affiliations see end of article.

Correspondence to Dr Kristina H Haugaa, Department of Cardiology, University of 0slo, Oslo 0424, Norway; Kristina.Haugaa@ medisin.uio.no

\section{ABSTRACT}

Objective Displacement of the mitral valve, mitral annulus disjunction (MAD), is described as a possible aetiology of sudden cardiac death. Stress-induced fibrosis in the mitral valve apparatus has been suggested as the underlying mechanism. We aimed to explore the association between stretch-related and fibrosis-related biomarkers and ventricular arrhythmias in MAD. We hypothesised that soluble suppression of tumourigenicity-2 (SST2) and transforming growth factor- $\beta 1$ (TGF $\beta 1$ ) are markers of ventricular arrhythmias in patients with MAD.

Methods We included patients with $\geq 1 \mathrm{~mm}$ MAD on cardiac MRI. We assessed left ventricular ejection fraction (LVEF) and fibrosis by late gadolinium enhancement (LGE). The occurrence of ventricular arrhythmia, defined as aborted cardiac arrest, sustained or non-sustained ventricular tachycardia, was retrospectively assessed. We assessed circulating SST2 and TGF $\beta 1$ levels.

Results We included 72 patients with MAD, of which $22(31 \%)$ had ventricular arrhythmias. Patients with ventricular arrhythmias had lower LVEF $(60 \%( \pm 6)$ vs $63 \%( \pm 6), p=0.04)$, more frequently papillary muscle fibrosis (14 (64\%) vs $10(20 \%), p<0.001)$ and higher sST2 levels $(31.6 \pm 10.1 \mathrm{ng} / \mathrm{mL}$ vs $25.3 \pm 9.2 \mathrm{ng} / \mathrm{mL}, \mathrm{p}=$ 0.01 ) compared with those without, while TGF $\beta 1$ levels did not differ $(p=0.29)$. Combining sST2 level, LVEF and papillary muscle fibrosis optimally detected individuals with arrhythmia (area under the curve $0.82,95 \% \mathrm{Cl} 0.73$ to 0.92$)$ and improved the risk model $(p<0.05)$ compared with single parameters.

Conclusion Circulating sST2 levels were higher in patients with MAD and ventricular arrhythmias compared with arrhythmia-free patients. Combining SST2, LVEF and LGE assessment improved risk stratification in patients with MAD.

\section{INTRODUCTION}

The increased risk for severe ventricular arrhythmias among patients with mitral valve prolapse (MVP) has been recognised for many years, with an estimated incidence rate of sudden cardiac death of $0.1 \%-0.4 \%$ per patient per year. ${ }^{12}$ Mitral annulus disjunction (MAD), alongside other factors such as the

\section{Key questions}

What is already known about this subject?

- Mitral annulus disjunction has been associated with an increased risk for ventricular arrhythmias, both in patients with and without concomitant mitral valve prolapse. Defining patients at high risk is challenging.

What does this study add?

- Our study is the first one to assess biomarkers in a patient cohort with mitral annulus disjunction. We demonstrated that soluble suppression of tumorigenicity-2 (SST2) levels were higher in patients with mitral annulus disjunction and ventricular arrhythmias, compared with those without arrhythmias. sST2 is released in the presence of myocardial stretch, and our findings might support the theory that mitral valve apparatus hypermobility causes stretch-induced arrhythmias.

How might this impact on clinical practice?

- A subset of patients with mitral annulus disjunction are susceptible to life-threatening ventricular arrhythmias. SST2 levels might help to identify highrisk individuals when combined with left ventricular ejection fraction and cardiac fibrosis. Future prospective studies are warranted to assess potential clinical implications of SST2: it could be integrated in a risk score, or rather serve as a screening tool, defining which patients with mitral annulus disjunction need further workup

presence of bileaflet MVP and focal fibrosis of the inferobasal left ventricular (LV) myocardium and papillary muscles, was described as an indicator of a more arrhythmogenic MVP phenotype. ${ }^{3-6}$ Recently, MAD has been associated with aborted cardiac arrest (ACA) and sustained ventricular tachycardia (VT), independent of the presence of MVP indicating MAD itself as an arrhythmogenic factor. ${ }^{7}$ MAD is a superior displacement of the mitral annulus away from the basal LV myocardium. ${ }^{8}$ The origin or development of 
MAD is not known, neither are the mechanisms causing ventricular arrhythmias. It has been hypothesised that MAD and redundant mitral leaflets might lead to a hypermobility of the mitral valve apparatus, subsequently causing increased stretch of the papillary muscles and the basal LV wall. ${ }^{3}$ Small myocardial lesions in association with increased mechanical stress could generate electrical instability, leading to life-threatening arrhythmias. ${ }^{349}$ Risk stratification in this population is challenging and no reliable markers for indicating primary prevention implantable cardioverter defibrillator are known. Biomarkers might help identifying patients with an increased risk for ventricular arrhythmias. Soluble suppression of tumorigenicity-2 (sST2) is a marker of myocardial stretch, ${ }^{10}$ while transforming growth factor- $\beta 1$ (TGF $\beta 1$ ) plays a key role in the development of replacement fibrosis and myxomatous mitral valve degeneration. ${ }^{11} 12$

The aim of our study was to analyse the presence of sST2 and TGF $\beta 1$ in patients with MAD. We hypothesised that these biomarkers, reflecting myocardial stretch and fibrosis, are markers of increased risk for ventricular arrhythmias.

\section{METHODS}

\section{Study population}

Patients were recruited between August 2015 and March 2018 in three centres: Oslo University Hospital Rikshospitalet, Norway, Drammen Hospital, Norway and Brussels University Hospital, Belgium. Cardiologists and sonographers at the three screening centres were educated to identify MAD by echocardiography. The potential study patients were invited to the study protocol including cardiac magnetic resonance (CMR), 24 hours ECG recording, ECG, blood sample acquisition and clinical examination. We included patients with a MAD of minimum $1 \mathrm{~mm}$ confirmed on CMR (online supplementary figure 1). Patients with established ischaemic, toxic or valvular heart disease other than degeneration of the mitral valve, or a history of mitral valve surgery or radiofrequency ablation of either VT or ventricular extrasystoles, were excluded. All study participants gave written informed consent.

\section{Arrhythmia detection}

Ventricular arrhythmia was defined as ACA, sustained VT or non-sustained VT (nsVT) ( $\geq 3$ consecutive ventricular beats at $\geq 150$ beats $/ \mathrm{min}$ ). We collected occurrence of arrhythmia from 24 hours ECG recording performed at inclusion and from previous exercise tests, 24 hours ECG recordings, telemetry, cardiac devices and medical records. We reported both current atrial fibrillation, present on the ECG performed at inclusion, and a history of atrial fibrillation, defined as a current or previously documented paroxysmal or permanent atrial fibrillation.

\section{Cardiac magnetic resonance}

CMR with cine imaging and late gadolinium enhancement (LGE) was performed using a $3 \mathrm{~T}$ unit (Ingenia,
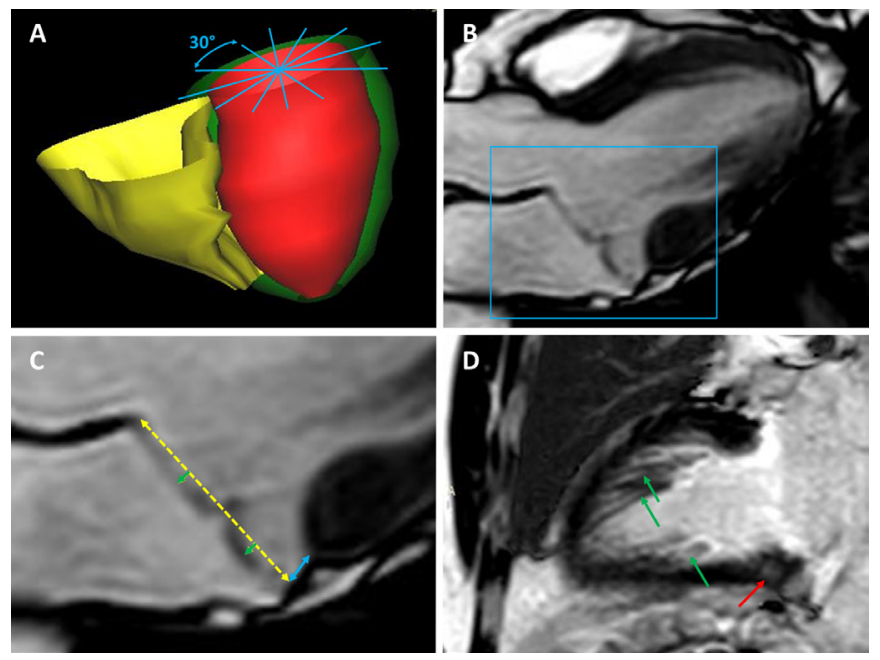

Figure 1 Cardiac magnetic resonance measurements. (A) Six long-axis images were obtained, with a $30^{\circ}$ angulation between each plane, as indicated by the blue lines. (B) Three-chamber view of a patient with mitral annulus disjunction (MAD), with prolapse of the mitral valve leaflets. (C) Zoom of (B): the yellow-dotted line represents the mitral annulus. The blue line represents the longitudinal MAD. This image shows the presence of prolapsing mitral leaflets, indicated by the two green arrows. (D) Late gadolinium enhancement on the basal left ventricular wall (red arrow) and the papillary muscles (green arrows).

Phillips Healthcare, Best, the Netherlands) and images were analysed using Segments V.1.9.30 (Medviso AB, Lund, Sweden) and Circle (Circle Cardiovascular Imaging, Calgary, Alberta, Canada), blinded to clinical data.

In patients with ACA, CMR was performed after a minimal period of 5 days and before ICD implantation. Attention was paid to exclude patients if postcardiac arrest myocardial stunning was observed.

Left ventricle end-systolic (LVESV) and end-diastolic volumes (LVEDV) were derived using the short-axis summation method and indexed for body surface area. ${ }^{13}$ Guided by a long-axis view, care was taken to identify the mitral annulus and include the basal sections of the $\mathrm{LV}$, but exclude prolapsed volume. LV ejection fraction (LVEF) was calculated by subtracting the LVESV from the LVEDV and dividing the difference by LVEDV. Mitral valve regurgitant volume (MRvol) was calculated by subtracting the aortic forward flow from the LV stroke volume or by subtracting right ventricular stroke volume from the LV stroke volume when aortic flow measurements were not available. ${ }^{14}$ Sphericity index was calculated by subtracting the LVEDV from the volume of a sphere with a diameter equal to the longitudinal diameter of the $\mathrm{LV}$ at end diastole. ${ }^{15}$

MVP was defined as an atrial displacement of the mitral leaflets of minimum $2 \mathrm{~mm}$ superior to the mitral annular plane during systole on a three-chamber view (figure 1C)..$^{1617}$

Longitudinal MAD was measured from the left atrial wall-mitral valve leaflet junction to the top of the LV wall 
during end systole on a three-chamber view (figure 1C). ${ }^{18}$ The circumferential MAD along the mitral annulus, expressed in degrees, was obtained by assessing the presence of MAD on six long-axis views, each separated by a $30^{\circ}$ angle (figure $\left.1 \mathrm{~A}\right) .^{7}$

LGE was obtained by acquiring short-axis slices covering the $\mathrm{LV}$ with a slice-to-slice increment of $10 \mathrm{~mm}, 10-20$ min after intravenous injection of gadopentetate dimeglumine. Supplemental long-axis images were acquired if LGE was suspected on short-axis images. The presence and location of LGE were visually assessed (figure 1D).

\section{Biomarker assessment}

The blood samples were acquired at inclusion and samples from all centres were analysed in one laboratory. sST2, TGF $\beta 1, N$-terminal probrain natriuretic peptide (NT-pro-BNP), C reactive protein (CRP) and creatinine were quantified. sST2 was analysed by enzyme immune assay using the Presage ST2 Assay (Critical Diagnostics, San Diego, California, USA). TGF $\beta 1$ was measured in duplicate using commercially available antibodies ( R\&D Systems, Minneapolis, Minnesota, USA) in a 384 format using the combination of a SELMA (Jena, Germany) pipetting robot and a BioTek (Winooski, Vermont, USA) dispenser/washer (EL406). Absorption was read at 450 $\mathrm{nm}$ with wavelength correction set to $540 \mathrm{~nm}$ (Synergy H1 Hybrid, Biotek, Vinooski, Vermont, USA). All intraassay coefficients of variation were $<6 \%$.

\section{Statistics}

Continuous data were presented as mean $\pm \mathrm{SD}$ or median and IQR. Categorical data were presented as number (\%). Comparisons were performed using Student's t-test, Mann-Whitney U test, $\chi 2$ or Fischer's exact test as appropriate. Correlations were assessed using Pearson or Spearman correlation coefficient as appropriate. Logistic regression analysis, adjusted for age and sex, was performed for parameters significant in univariate analyses $(p<0.05)$. Receiver operating characteristics (ROC) curve analyses were performed for significant parameters $(p<0.05)$ from unadjusted univariate logistic regression, as well as for a risk model combining these parameters. The areas under the curves (AUC) of ROC curves were compared. Statistical analysis was performed using SPSS

Patients with MAD $\geq 1 \mathrm{~mm}$ on $C M R$ with contrast and biomarkers assessment, $\mathrm{N}=79$

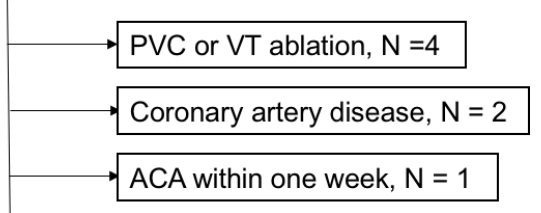

Patients included, $\mathrm{N}=72$

Figure 2 Inclusion flow chart. ACA, aborted cardiac arrest; $\mathrm{CMR}$, cardiac magnetic resonance; MAD, mitral annulus disjunction; PVC, premature ventricular contractions; VT, ventricular tachycardia.
V.24.0 and risk model comparisons by Stata/SE V.15.1 (StataCorp LLC).

\section{RESULTS}

\section{Patient characteristics}

We included 72 patients with MAD who underwent both biomarker assessment and CMR imaging with LGE (figure 2). The majority of the patients were women $(67 \%)$ and the median age was 55 (35-62) years old (table 1).

A total of $22(31 \%)$ patients had experienced ventricular arrhythmias, of which $18(82 \%)$ had a history of arrhythmia before inclusion (5 (23\%) ACA, and 13 $(59 \%)$ nsVT). Fifty-six (78\%) patients had a 24 hours ECG recording at inclusion, which revealed nsVT in another 4 (18\%) patients. One patient, who had his blood sample taken within 1 week after ACA, was excluded to avoid possible postreanimation changes in biomarkers.

Most patients were in NYHA functional class I, regardless of their arrhythmic status $(19(86 \%)$ in the VA group vs $42(84 \%)$ in the non-VA group, $\mathrm{p}=0.80)$.

\section{Cardiac magnetic resonance}

LVEF was significantly lower $(60 \%( \pm 6)$ vs $63 \%( \pm 6)$, $\mathrm{p}=0.04)$, though still in the normal range, in patients with ventricular arrhythmias (table 2). The majority of the patients $(83 \%)$ presented with no or mild mitral regurgitation, and mitral regurgitation severity did not differ between the arrhythmic and the non-arrhythmic group (table 2). LGE prevalence was higher in patients with ventricular arrhythmias compared with non-arrhythmic patients, particularly at the level of the papillary muscles (14 (64\%) vs $10(20 \%), \mathrm{p}<0.001)$.

\section{Biomarkers SST2 and TGF $\beta 1$}

The concentration of sST2 was higher in patients with ventricular arrhythmias compared with those without $(31.6 \pm 10.1 \mathrm{ng} / \mathrm{mL}$ vs $25.3 \pm 9.2 \mathrm{ng} / \mathrm{mL}, \mathrm{p}=0.01) \quad$ (table 2 , figure 3). A cut-off value of sST2 of $26 \mathrm{ng} / \mathrm{mL}$ differentiated patients with and without VA with a $68 \%$ sensitivity and a $58 \%$ specificity. Relatively less women had sST2 levels above this cut-off value $(19(53 \%)$ vs $29(81 \%)$, $\mathrm{p}=0.01$ ) (table 1). There was a weak correlation between sST2 and both LVEDV ( $\mathrm{r}=0.26, \mathrm{p}=0.03)$ and LVESV $(\mathrm{r}=0.25, \mathrm{p}=0.03)$, but no association between sST2 and indexed volumes nor between sST2 and MRvol.

There were no differences in circulating concentrations of TGF $\beta 1$ among patients with and without arrhythmias $(2.59 \mathrm{ng} / \mathrm{mL}(1.86-4.64)$ vs $2.21 \mathrm{ng} / \mathrm{mL}(1.70-3.90)$, $\mathrm{p}=0.29$ ) (figure $3 \mathrm{~A}$ ). Circulating TGF $\beta 1$ levels were markedly higher in patients with LGE compared with patients without LGE $(3.35 \mathrm{ng} / \mathrm{mL}(2.21-6.64)$ vs $1.89 \mathrm{ng} / \mathrm{mL}$ (1.45-2.35), $\mathrm{p}<0.001)$. Patients with circumferential MAD $>180^{\circ}$ had significantly higher circulating TGF $\beta 1$ levels (4.20 ng/mL (2.01-8.53) vs $2.02 \mathrm{ng} / \mathrm{mL}$ (1.64-2.99), $\mathrm{p}=0.003$ ) and TGF $\beta 1$ levels correlated with the extent of the circumferential MAD $(r=0.45, p<0.001)$. 
Table 1 Clinical characteristics and cardiac magnetic resonance measurements of 72 patients with mitral annulus disjunction (MAD), dichotomised by soluble suppression of tumourigenicity-2 (sST2) levels $<$ or $\geq$ the optimal cut-off value

\begin{tabular}{|c|c|c|c|c|}
\hline & Total $(n=72)$ & sST2 <26 ng/mL (n=36) & $\mathrm{sST} 2 \geq 26 \mathrm{ng} / \mathrm{mL}(\mathrm{n}=36)$ & $P$ value \\
\hline \multicolumn{5}{|l|}{ Clinical characteristics } \\
\hline Age, years (IQR) & $55(35-62)$ & $52(34-61)$ & $58(25-64)$ & 0.65 \\
\hline Female, $n(\%)$ & $48(67)$ & $29(81)$ & $19(53)$ & 0.01 \\
\hline Body mass index, kg/m² ( $\pm \mathrm{SD})$ & $22.6( \pm 3.1)$ & $22.5( \pm 3.4)$ & $22.7( \pm 2.8)$ & 0.77 \\
\hline NYHA functional class (IQR) & $1(1-1)$ & $1(1-1)$ & $1(1-1)$ & 0.36 \\
\hline Atrial fibrillationt, $\mathrm{n}(\%)$ & $5(7)$ & $1(3)$ & $4(11)$ & 0.36 \\
\hline Ventricular arrhythmia, $\mathrm{n}(\%)$ & $22(31)$ & $7(19)$ & $15(42)$ & 0.04 \\
\hline \multicolumn{5}{|l|}{ Cardiac magnetic resonance } \\
\hline LVEDVi, $\mathrm{mL} / \mathrm{m}^{2}( \pm \mathrm{SD})$ & $85( \pm 19)$ & $82( \pm 17)$ & $87( \pm 20)$ & 0.24 \\
\hline LVESVi, $\mathrm{mL} / \mathrm{m}^{2}\left({ }^{( \pm \mathrm{SD})}\right.$ & $32( \pm 10)$ & $31( \pm 9)$ & $34( \pm 10)$ & 0.17 \\
\hline LVEF, \% ( \pm SD) & $62( \pm 6)$ & $63( \pm 6)$ & $61( \pm 7)$ & 0.24 \\
\hline Mitral regurgitant volume ${ }^{\star}, \mathrm{mL}$ (IQR) & $17(9-26)$ & $20(8-25)$ & $16(10-28)$ & 0.44 \\
\hline MVP present, $n(\%)$ & $52(72)$ & $25(69)$ & $27(75)$ & 0.60 \\
\hline Longitudinal MAD on $3 \mathrm{CH}, \mathrm{mm}$ (IQR) & $2(0-6)$ & $2(0-6)$ & $3(0-5)$ & 0.92 \\
\hline Circumferential MAD, ${ }^{\circ}(\mathrm{IQR})$ & $180(120-210)$ & $180(120-210)$ & $180(120-210)$ & 0.48 \\
\hline LGE present, n (\%) & $36(50)$ & $18(50)$ & $18(50)$ & 1.00 \\
\hline LGE in LV myocardium, n (\%) & $21(29)$ & $11(31)$ & $10(28)$ & 0.80 \\
\hline LGE in papillary muscles, n (\%) & $24(33)$ & $10(28)$ & $14(39)$ & 0.32 \\
\hline
\end{tabular}

The optimal cut-off value was defined by receiver operating characteristics curve analysis. The $p$ values are calculated by Student's t-test, Mann-Whitney $U$ test, $\chi^{2}$ test, or Fischer's exact test as appropriate.

${ }^{*}$ Aortic forward flow was available in $21(29 \%)$ patients.

$\dagger$ Atrial fibrillation present at the time of inclusion.

LGE, late gadolinium enhancement; LVEDVi, left ventricular end-diastolic volume indexed for body surface area; LVEF, left ventricular ejection fraction; LVESVi, left ventricular end-systolic volume indexed for body surface area; MR, mitral regurgitation; MVP, mitral valve prolapse; NYHA, New York Heart Association.

\section{Risk markers for ventricular arrhythmias}

Both LVEF and sST2 showed added value on top of papillary muscle LGE to discriminate patients with and without ventricular arrhythmias (online supplementary figure 2). A risk model comprising LVEF, sST2 levels and LGE at the papillary muscles had a higher AUC than each of these parameters alone for the identification of patients with ventricular arrhythmias (figure 4). This combined model also performed well to identify patients with ACA only (ROC curve AUC 0.83 (95\% CI 0.72 to 0.95 )).

\section{DISCUSSION}

This is the first study showing the association between biomarkers and ventricular arrhythmias in patients with MAD. Patients with MAD with ventricular arrhythmias had higher levels of circulating sST2 compared with the arrhythmia free, indicating that myocardial stretch may be a mechanism in MAD arrhythmogenesis. The combination of higher sST2, lower LVEF and presence of papillary muscle fibrosis improved the risk stratification model for ventricular arrhythmias in patients with MAD and may be of clinical importance in identifying high-risk individuals. While TGF $\beta 1$ was not associated with ventricular arrhythmias, higher levels of TGF $\beta 1$ were associated with myocardial and papillary muscle fibrosis and a larger circumferential MAD.

SST2 IN PATIENTS WITH MAD

sST2 is an interleukin 1 receptor family member, that is secreted by cardiac fibroblasts and cardiomyocytes in circumstances of biomechanical stress. ${ }^{10} 19$ While the interaction between interleukin 33 (IL-33) and membrane-bound ST2 (ST2L) has cardioprotective effects by reducing cardiac fibrosis and hypertrophy, sST2 acts as a decoy receptor by binding free IL-33 and limiting IL-33/ST2L interaction. ${ }^{19}$

In our study, higher levels of sST2 were associated with ventricular arrhythmias. These findings may indicate that stretch mechanisms are involved in arrhythmogenesis, possibly due to the altered anatomy and function of the mitral valve apparatus. The hypermobility of the mitral valve apparatus in patients with MAD is thought to be an important element in the pathophysiological process leading to arrhythmias in these patients. ${ }^{3}{ }^{46}$ The higher sST2 levels in arrhythmic MAD patients in our study might be a consequence of elevated mechanical stress exerted on the basal LV wall and the papillary muscles. Previously, deformation imaging in patients with MVP has shown increased prestretch and postsystolic shortening 
Table 2 Clinical characteristics, cardiac magnetic resonance measurements and biomarker levels of 72 patients with mitral annulus disjunction, dichotomised in ventricular arrhythmia (VA) $(n=22)$ and no VA $(n=50)$

\begin{tabular}{|c|c|c|c|c|c|}
\hline & No VA $(n=50)$ & VA $(n=22)$ & $P$ value & OR $(95 \% \mathrm{Cl})$ & $P$ value \\
\hline \multicolumn{6}{|l|}{ Clinical } \\
\hline Age, years (IQR) & $57(35-63)$ & $48(32-61)$ & 0.35 & & \\
\hline Female, n (\%) & $33(66)$ & $15(68)$ & 0.86 & & \\
\hline NYHA functional class, (IQR) & $1(1-1)$ & $1(1-1)$ & 0.88 & & \\
\hline Atrial fibrillation§, n (\%) & 7 (14) & $3(14)$ & 1.00 & & \\
\hline \multicolumn{6}{|l|}{ CMR } \\
\hline LVEF, \% ( $( \pm \mathrm{SD})$ & $63( \pm 6)$ & $60( \pm 6)$ & 0.04 & $0.64^{*}(0.41$ to 1.05$)$ & 0.05 \\
\hline LVEDVi, $\mathrm{mL} / \mathrm{m}^{2}( \pm \mathrm{SD})$ & $84( \pm 19)$ & $87( \pm 19)$ & 0.44 & & \\
\hline LVESVi, $\mathrm{mL} / \mathrm{m}^{2}( \pm \mathrm{SD})$ & $31( \pm 10)$ & $35( \pm 9)$ & 0.09 & & \\
\hline Mitral regurgitant volume, $\mathrm{mL}$ (IQR) & $21(9-26)$ & $14(7-27)$ & 0.70 & & \\
\hline No or mild MR, $\mathrm{n}(\%)$ & $42(84)$ & $18(82)$ & 0.82 & & \\
\hline Moderate MR, n (\%) & $7(14)$ & $4(18)$ & 0.73 & & \\
\hline Severe MR, n (\%) & $1(2)$ & $0(0)$ & 1.00 & & \\
\hline Bileaflet MVP, n (\%) & $22(44)$ & $9(41)$ & 0.81 & & \\
\hline Spheriticy indext, (IQR) & $0.28(0.26-0.32)$ & $0.28(0.20-0.34)$ & 0.66 & & \\
\hline LGE in LV myocardium, n (\%) & $13(26)$ & $8(36)$ & 0.37 & & \\
\hline LGE in papillary muscles, $n(\%)$ & $10(20)$ & $14(64)$ & $<0.001$ & 7.26 (2.34 to 22.53$)$ & 0.001 \\
\hline \multicolumn{6}{|l|}{ Biomarkers } \\
\hline sST2, ng/mL ( \pm SD) & $25.3( \pm 9.2)$ & $31.6( \pm 10.1)$ & 0.01 & $1.52 \ddagger(1.10$ to 2.06$)$ & 0.01 \\
\hline TGF $\beta 1, n g / m L ~(I Q R)$ & $2.21(1.70-3.98)$ & $2.59(1.86-4.64)$ & 0.29 & & \\
\hline NT-pro-BNP, pmol/L (IQR) & $9.7(5.6-22.5)$ & $14.5(7.0-42.4)$ & 0.31 & & \\
\hline CRP, mg/L (IQR) & $0.7(0.0-2.1)$ & $0.9(0.0-1.7)$ & 0.62 & & \\
\hline Creatinine, $\mu \mathrm{mol} / \mathrm{L}( \pm \mathrm{SD})$ & $72( \pm 14)$ & $76( \pm 15)$ & 0.40 & & \\
\hline
\end{tabular}

The $p$ values are calculated by Student's t-test, Mann-Whitney $U$ test, Fisher's exact test or $\chi^{2}$ test as appropriate. OR for the occurrence of $\mathrm{VA}$, adjusted for age and sex, was calculated by logistic regression analysis.

*OR per $5 \%$ increment of LVEF.

†Measured in 51 patients (no VA $n=35$ and VA $n=16$ ).

†OR per $5 \mathrm{ng} / \mathrm{mL}$ increment of sST2.

$\S$ Combination of a history of and current paroxysmal or permanent atrial fibrillation.

CMR, cardiac magnetic resonance; CRP, C reactive protein; LGE, late gadolinium enhancement; LVEDVi, left ventricular end-diastolic volume indexed for body surface area; LVEF, left ventricular ejection fraction; LVESVi, left ventricular end-systolic volume indexed for body surface area; MR, mitral regurgitation; MVP, mitral valve prolapse; NT-pro-BNP, N-terminal probrain natriuretic peptide;sST2, soluble suppression of tumourigenicity-2.

in basal segments and segments adjacent to papillary muscle insertion. ${ }^{20}$ The stretch on the subvalvular apparatus could generate premature ventricular contractions and might trigger potentially fatal VA.

The theory of arrhythmogenicity of the hypermobile mitral valve apparatus is supported by the discovery of fibrotic scars on the papillary muscles and basal LV wall, concordant with the origin of PVCs in the arrhythmic patient population. ${ }^{21}{ }^{22}$ Mitral valve repair and subsequent reduction of the hypermobility led to reduction of ventricular arrhythmias in several studies. ${ }^{23}$ Those studies, as opposed to ours, were performed in patients with severe mitral regurgitation. In our study, ventricular arrhythmias occurred independently of mitral regurgitation severity, concordant with early data. ${ }^{12}$ This is an important clinical message and should encourage the clinician to evaluate arrhythmic risk in patients with MAD, independent of MVP and severity of mitral regurgitation.

Increased levels of sST2 have previously been associated with ventricular arrhythmias in patients with arrhythmogenic and hypertrophic cardiomyopathy. ${ }^{24} 25$ These reports are in line with our findings and support the idea of MAD as a cardiomyopathic disorder. However, the exact pathophysiological mechanisms linking sST2 to arrhythmogenesis in cardiomyopathies are not explored and need further investigations.

Furthermore, sST2 predicted mortality in patients with heart failure in a previous report. ${ }^{26}$ Although none of our patients had overt heart failure, LVEF was lower in patients with ventricular arrhythmias compared with those without. This might reflect the presence of underlying interstitial fibrosis, as previously seen on 


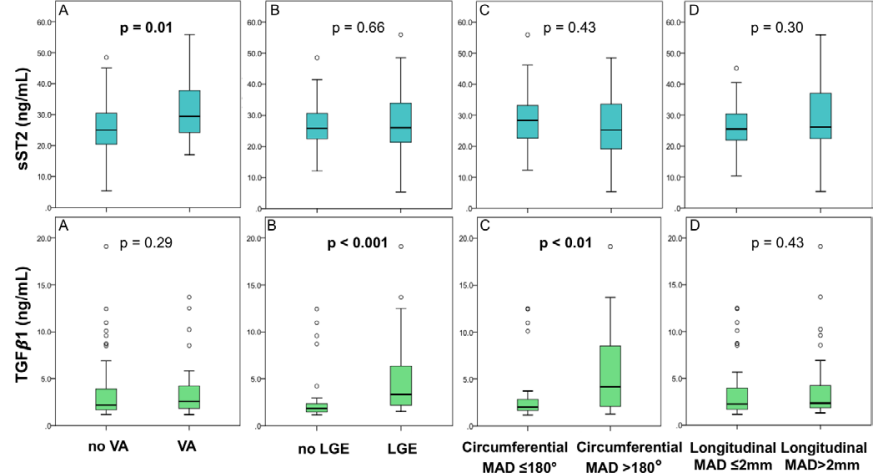

Figure 3 Boxplots representing soluble suppression of tumourigenicity-2 (sST2) levels in the upper row and transforming growth factor- $\beta 1$ (TGF $\beta 1$ ) levels in the lower row. (A) No severe ventricular arrhythmia (VA) versus severe VA. (B) No late gadolinium enhancement (LGE) present on cardiac magnetic resonance (CMR) versus LGE present on CMR. (C) Circumferential mitral annulus disjunction (MAD) $\leq 180^{\circ}$ versus circumferential MAD $>180^{\circ}$. (D) Longitudinal $M A D$ on the three-chamber view $\leq 2 \mathrm{~mm}$ versus longitudinal MAD on the three-chamber view $>2 \mathrm{~mm}$. $P$ values were obtained by performing Student's t-test for SST2 and MannWhitney $U$ test for TGF $\beta 1$.

the postmortem analysis of patients with MVP who died suddenly. ${ }^{27}$ When LVEF, sST2 levels and LGE at the papillary muscle level were combined to form a risk model, there was an improvement of the ability to discriminate patients with arrhythmia, compared with using the presence of LGE or LVEF alone. Considering the complexity of the arrhythmogenic process and the absence of a single obvious risk marker, risk stratification combining several parameters is most likely to eventually improve the prediction of life-threatening arrhythmias in patients with MAD.

\section{TGF $\beta 1$ in patients with MAD}

The cytokine TGF $\beta 1$ plays an important role in the development of myxomatous mitral valve disease. Genetic,
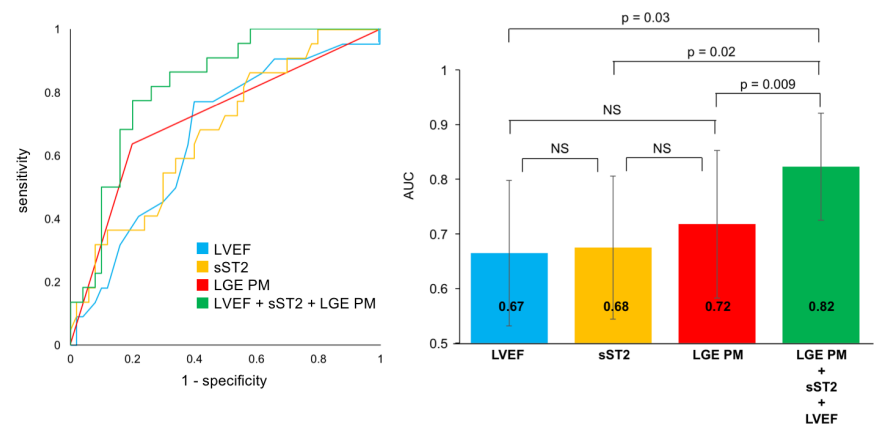

Figure 4 Receiver operating characteristics (ROC) curves for the differentiation of patients with and without ventricular arrhythmias. ROC curve for left ventricular ejection fraction (LVEF) (blue), soluble suppression of tumourigenicity-2 (sST2) levels (yellow), late gadolinium enhancement at the papillary muscles (LGE PM) (red) and the combined model of these three parameters (green) in 72 patients with mitral annulus disjunction (left panel) and comparisons of the ROC curves' area under the curve (AUC) (right panel). NS, not significant. biological and mechanical factors can increase the expression of TGF $\beta 1$ in the mitral valve, stimulating the differentiation of endothelial and interstitial cells into extracellular matrix producing myofibroblasts. ${ }^{28} 29$ The pathophysiological process resulting in MAD is still unknown, but one might hypothesise that it involves TGF $\beta 1$-pathway activation, similar to the process of mitral valve degeneration. This might explain why a wider circumferential MAD around the mitral ring was associated with higher levels of circulating TGF 31 . Longitudinal MAD, measured at one single location around the circumference, was not associated with TGF $\beta 1$. MAD can be present partially along the mitral ring, interspersed with non-disjunctive annulus. ${ }^{730}$ The circumferential extent of MAD might therefore better represent the extent of the affection than a single longitudinal MAD measurement and explain the better correlation with TGF $\beta 1$ levels.

TGF $\beta 1$ is also a key player in tissue healing and fibrotic scar formation. ${ }^{12}$ This might explain why TGF $\beta 1$ levels were higher in patients with myocardial or papillary muscle replacement fibrosis, detected by LGE on CMR. Small myocardial tears, caused by repeated mechanical stress, may activate the TGF $\beta 1$ pathway, resulting in small areas of replacement fibrosis as seen on CMR with contrast.

\section{Limitations}

The patients were included prospectively and the occurrence of ventricular arrhythmias was assessed retrospectively. A prospective study is warranted for the analysis of the predictive power of sST2 to identify patients with MAD with an increased risk for life-threatening ventricular arrhythmias. Although multicentric, our study included a relatively small group of patients and events were limited.

Circulating sST2 and TGF $\beta 1$ levels reflect contribution from multiple sources, including but not exclusively the heart. Thus, other conditions might influence circulating biomarker levels. However, CRP and NT-pro-BNP levels were within normal ranges in all patients, suggesting that neither inflammation nor general LV overload caused the differences in sST2 and TBF $\beta 1$. Cardiac tissue biopsy and gene expression analysis might better represent the local mechanics but is invasive and not clinically applicable.

We did not assess troponin levels in our study. This might be considered for future studies.

\section{Future perspectives}

The occurrence of life-threatening ventricular arrhythmias in patients with MAD, whether associated with the presence of MVP or not, is of great concern and many questions regarding pathophysiology and risk stratification remain unanswered. ${ }^{34}$

In our study, 24 hours ECG recording at inclusion revealed nsVT in some patients. Whether 24 hours ECG recording should be performed in every patient with MAD is unclear. Prospective studies should elucidate if 24 hours ECG recording derived parameters such as 
frequent premature ventricular complexes or nsVT are markers of later life-threatening ventricular arrhythmias.

The biomarker sST2, which is induced by myocardial stretch, ${ }^{10} 19$ might be a marker for the arrhythmic risk in this population. The maximal distance between the papillary muscles and the mitral leaflets may be a surrogate marker for local stretch and future studies should foresee dedicated images to allow this measurement. A prospective study including a large cohort of patients with MAD should clarify the prognosis of this population and allow elaboration of a risk stratification strategy.

\section{CONCLUSIONS}

Higher circulating sST2 levels were associated with ventricular arrhythmias in patients with MAD, while TGF $\beta 1$ levels were not. Combining the assessment of sST2 levels, LVEF and the presence of LGE at the level of the papillary muscles resulted in excellent detection of high-risk individuals. Applying this combined risk model might improve risk stratification for severe ventricular arrhythmias in patients with MAD.

\author{
Author affiliations \\ ${ }^{1}$ Geneeskunde en farmacie, Vrije Universiteit Brussel, Brussel, Belgium \\ ${ }^{2}$ Department of Cardiology, Oslo University Hospital, Rikshospitalet, Oslo, Norway \\ ${ }^{3}$ Centrum voor Hart- en Vaatziekten, UZ Brussel, Brussels, Belgium \\ ${ }^{4}$ Institute for Clinical Medicine, Universitetet i Oslo, Oslo, Norway \\ ${ }^{5}$ Department of Radiology and Nuclear Medicine, Oslo University Hospital, \\ Rikshospitalet, Oslo, Norway \\ ${ }^{6}$ The Intervention Centre, Oslo universitetssykehus Rikshospitalet, 0slo, Norway \\ ${ }^{7}$ Radiology (MRI), Uz Brussel, Jette, Belgium \\ ${ }^{8}$ Research Institute of Internal Medicine, Oslo Universisty Hospital, Rikshospitalet, \\ Oslo, Norway \\ ${ }^{9}$ Academic Medical Center, University of Amsterdam, Amsterdam, Netherlands
}

Acknowledgements The authors would like to thank Johan de Mey and Hubert Raeymaekers from the Department of Radiology at University Hospital Brussels for their support.

Contributors ES, LAD, ES, ØHL, TE, SD, BC and KHH: conception and design of work; acquisition, analysis and interpretation of data; drafting and revising the manuscript critically for important intellectual content; final approval of the manuscript submitted; he/she is accountable for all aspects of the work, in ensuring that questions related to the accuracy or integrity of any part of the work are appropriately investigated and resolved. AM, EH, KT, TU, MR and CC: acquisition, analysis and interpretation of data; drafting and revising the manuscript critically for important intellectual content; final approval of the manuscript submitted; he/ she is accountable for all aspects of the work, in ensuring that questions related to the accuracy or integrity of any part of the work are appropriately investigated and resolved.

Funding This work was supported by a public grant [203489/030] from the Norwegian Research Council, Oslo, Norway. Esther Scheirlynck received a 2018 European Society of Cardiology research grant.

Competing interests None declared.

Patient consent for publication Not required.

Ethics approval The study complied with the Declaration of Helsinki and the research protocol was approved by the regional ethical committees.

Provenance and peer review Not commissioned; externally peer reviewed.

Data sharing statement All data relevant to the study are included in the article or uploaded as supplementary information.

Open access This is an open access article distributed in accordance with the Creative Commons Attribution Non Commercial (CC BY-NC 4.0) license, which permits others to distribute, remix, adapt, build upon this work non-commercially, and license their derivative works on different terms, provided the original work is properly cited, appropriate credit is given, any changes made indicated, and the use is non-commercial. See: http://creativecommons.org/licenses/by-nc/4.0/.

\section{REFERENCES}

1. Zuppiroli A, Rinaldi M, Kramer-Fox R, et al. Natural history of mitral valve prolapse. Am J Cardiol 1995;75:1028-32.

2. Nishimura RA, McGoon MD, Shub C, et al. Echocardiographically documented mitral-valve prolapse. Long-term follow-up of 237 patients. N Engl J Med 1985;313:1305-9.

3. Perazzolo Marra M, Basso C, De Lazzari M, et al. Morphofunctional abnormalities of mitral annulus and arrhythmic mitral valve prolapse. Circ Cardiovasc Imaging 2016;9:e005030.

4. Basso C, Perazzolo Marra M, Rizzo S, et al. Arrhythmic mitral valve prolapse and sudden cardiac death. Circulation 2015;132:556-66.

5. Sriram CS, Syed FF, Ferguson ME, et al. Malignant bileaflet mitral valve prolapse syndrome in patients with otherwise idiopathic outof-hospital cardiac arrest. J Am Coll Cardiol 2013;62:222-30.

6. Sheppard MN, Steriotis AK, Sharma S, et al. Letter by Sheppard et al Regarding Article, "Arrhythmic Mitral Valve Prolapse and Sudden Cardiac Death". Circulation 2016;133:e458.

7. Dejgaard LA, Skjølsvik ET, Lie Øyvind $\mathrm{H}$, et al. The mitral annulus disjunction arrhythmic syndrome. J Am Coll Cardiol 2018;72:1600-9.

8. Hutchins GM, Moore GW, Skoog DK. The association of floppy mitral valve with disjunction of the mitral annulus fibrosus. $N$ Engl $J$ Med 1986;314:535-40.

9. Lancellotti P, Garbi M. Malignant mitral valve prolapse: substrates to ventricular remodeling and arrhythmias. Circ Cardiovasc Imaging 2016;9:e005248.

10. Weinberg EO, Shimpo M, De Keulenaer GW, et al. Expression and regulation of ST2, an interleukin-1 receptor family member, in cardiomyocytes and myocardial infarction. Circulation 2002;106:2961-6.

11. Delling FN, Vasan RS. Epidemiology and pathophysiology of mitral valve prolapse: new insights into disease progression, genetics, and molecular basis. Circulation 2014;129:2158-70.

12. Border WA, Noble NA. Transforming growth factor beta in tissue fibrosis. N Engl J Med 1994;331:1286-92.

13. Schulz-Menger J, Bluemke DA, Bremerich J, et al. Standardized image interpretation and post processing in cardiovascular magnetic resonance: Society for cardiovascular magnetic resonance (SCMR) Board of trustees Task Force on standardized post processing. $J$ Cardiovasc Magn Reson 2013;15.

14. Krieger EV, Lee J, Branch KR, et al. Quantitation of mitral regurgitation with cardiac magnetic resonance imaging: a systematic review. Heart 2016;102:1864-70.

15. Mannaerts HFJ, van der Heide JA, Kamp O, et al. Early identification of left ventricular remodelling after myocardial infarction, assessed by transthoracic 3D echocardiography. Eur Heart J 2004;25:680-7.

16. Zoghbi WA, Adams D, Bonow RO, et al. Recommendations for noninvasive evaluation of native valvular regurgitation: a report from the American Society of echocardiography developed in collaboration with the Society for cardiovascular magnetic resonance. J Am Soc Echocardiogr 2017;30:303-71.

17. Freed LA, Levy D, Levine RA, et al. Prevalence and clinical outcome of mitral-valve prolapse. N Engl J Med 1999;341:1-7.

18. Carmo P, Andrade MJ, Aguiar C, et al. Mitral annular disjunction in myxomatous mitral valve disease: a relevant abnormality recognizable by transthoracic echocardiography. Cardiovasc Ultrasound 2010;8.

19. Sanada S, Hakuno D, Higgins LJ, et al. IL-33 and ST2 comprise a critical biomechanically induced and cardioprotective signaling system. J Clin Invest 2007;117:1538-49.

20. Huttin O, Pierre S, Venner $\mathrm{C}$, et al. Interactions between mitral valve and left ventricle analysed by 2D speckle tracking in patients with mitral valve prolapse: one more piece to the puzzle. Eur Heart $J$ Cardiovasc Imaging 2016.

21. Fulton BL, Liang JJ, Enriquez A, et al. Imaging characteristics of papillary muscle site of origin of ventricular arrhythmias in patients with mitral valve prolapse. $J$ Cardiovasc Electrophysiol 2018;29:146-53.

22. Syed FF, Ackerman MJ, McLeod CJ, et al. Sites of successful ventricular fibrillation ablation in bileaflet mitral valve prolapse syndrome. Circ Arrhythm Electrophysiol 2016;9.

23. Vaidya VR, DeSimone CV, Damle N, et al. Reduction in malignant ventricular arrhythmia and appropriate shocks following surgical correction of bileaflet mitral valve prolapse. J Interv Card Electrophysiol 2016;46:137-43. 
24. Broch K, Leren IS, Saberniak J, et al. Soluble ST2 is associated with disease severity in arrhythmogenic right ventricular cardiomyopathy. Biomarkers 2017;22:367-71.

25. Gawor M, Śpiewak M, Janas J, et al. The usefulness of SST2 and galectin-3 as novel biomarkers for better risk stratification in hypertrophic cardiomyopathy. Kardiol Pol 2017;75:997-1004

26. Aimo A, Vergaro G, Passino C, et al. Prognostic value of soluble suppression of Tumorigenicity-2 in chronic heart failure: a metaanalysis. JACC Heart Fail 2017:5:280-6.

27. Garbi M, Lancellotti P, Sheppard MN. Mitral valve and left ventricular features in malignant mitral valve prolapse. Open Heart 2018;5:e000925.
28. Hagler MA, Hadley TM, Zhang H, et al. TGF- $\beta$ signalling and reactive oxygen species drive fibrosis and matrix remodelling in myxomatous mitral valves. Cardiovasc Res 2013;99:175-84.

29. Rizzo S, Basso C, Lazzarini E, et al. TGF-beta1 pathway activation and adherens junction molecular pattern in nonsyndromic mitral valve prolapse. Cardiovasc Pathol 2015;24:359-67.

30. Angelini A, Ho SY, Anderson RH, et al. A histological study of the atrioventricular junction in hearts with normal and prolapsed leaflets of the mitral valve. Br Heart J 1988;59:712-6. 